\title{
Analysis of Molecular Interactions between Components in Phospholipid-Immunosuppressant-Antioxidant Mixed Langmuir Films
}

\author{
Małgorzata Jurak, ${ }^{\text {a* }}$ Klaudia Szafran ${ }^{\mathrm{a}}$, Pilar Cea ${ }^{\mathrm{b}, \mathrm{c}}$ and Santiago Martín ${ }^{\mathrm{b}, \mathrm{c}}$ \\ a Department of Interfacial Phenomena, Institute of Chemical Sciences, Faculty of Chemistry, \\ Maria Curie-Skłodowska University in Lublin, 20031 Lublin, Poland \\ ${ }^{b}$ Instituto de Nanociencia y Materiales de Aragón (INMA), CSIC-Universidad de Zaragoza, \\ 50009 Zaragoza, Spain \\ c Departamento de Química Física, Facultad de Ciencias, Universidad de Zaragoza, \\ 50009 Zaragoza, Spain \\ *Corresponding author (M. Jurak) \\ e-mail address: malgorzata.jurak@umcs.pl \\ phone: +48815375547 , fax: +48815375656
}

\section{SUPPORTING INFORMATION}

Figure S1. Compression modulus $\left(C_{S}^{-1}\right)$ versus surface pressure $(\pi)$ for the binary and ternary monolayers.

Figure S2. Surface pressure, $\pi-$, electric surface potential, $\Delta V-$, and apparent dipole moment, $\mu_{a}-$, area, $A$, isotherms for all monolayers studied. 

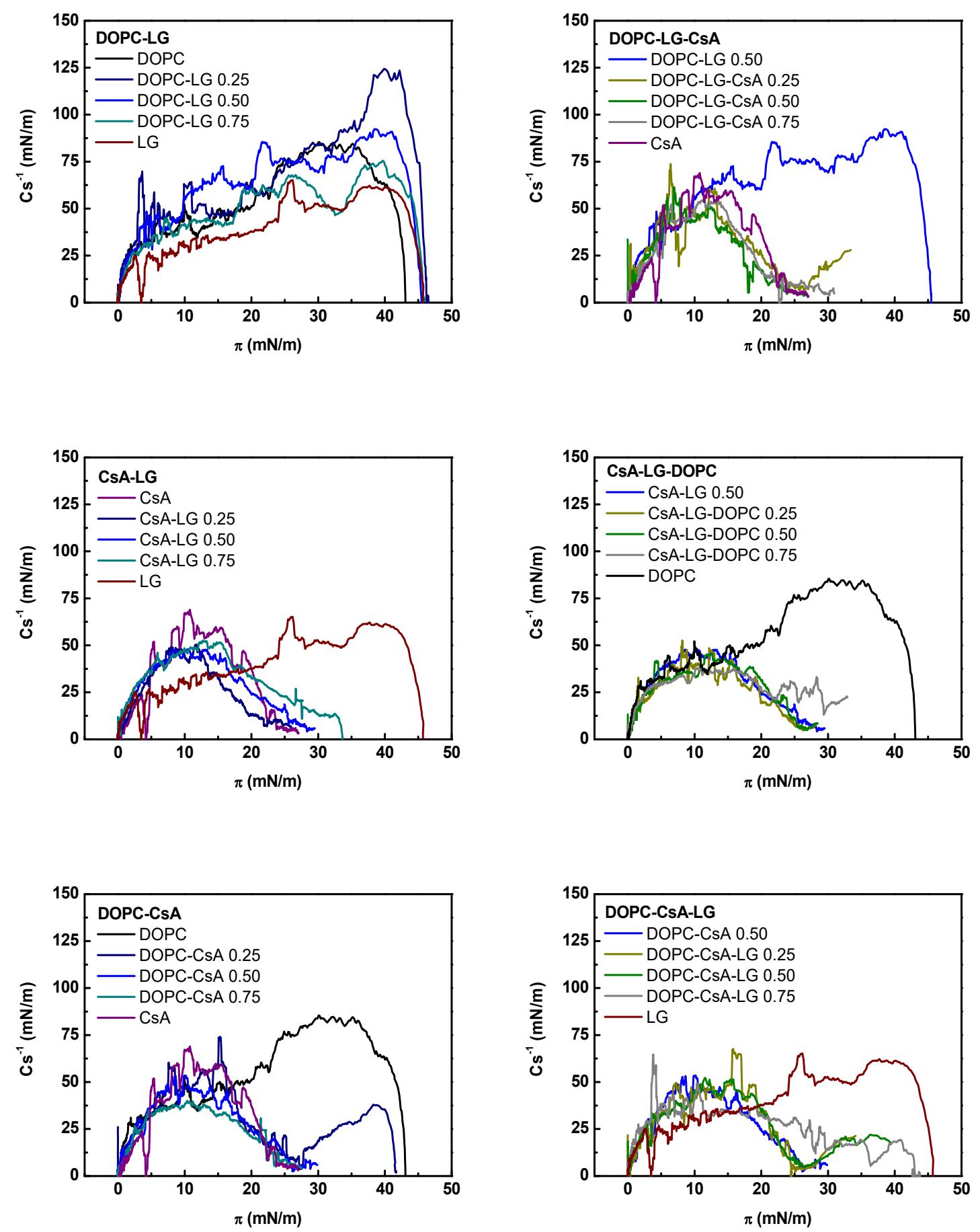

Figure S1. Compression modulus $\left(C_{S}^{-1}\right)$ versus surface pressure $(\pi)$ for the binary and ternary monolayers. 


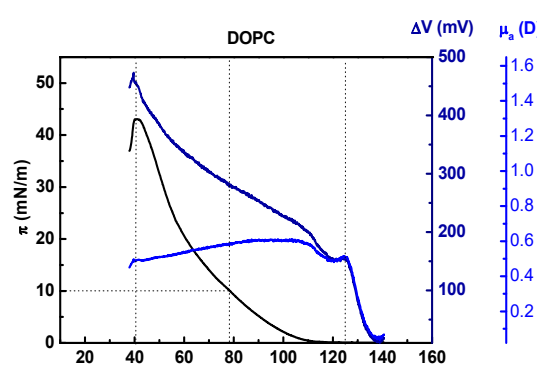

$A\left(\AA^{2} /\right.$ molecule $)$

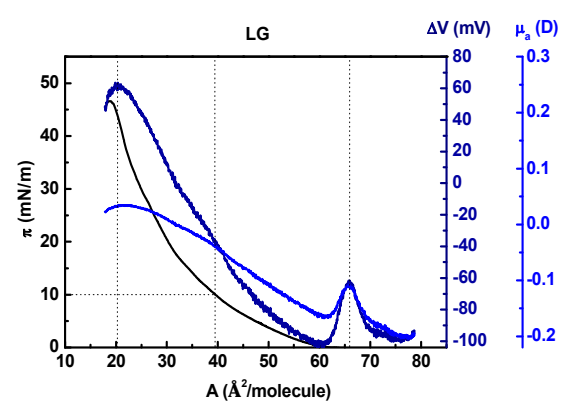

CSA $\quad \Delta V(m V) \quad \mu_{3}(\mathrm{D})$

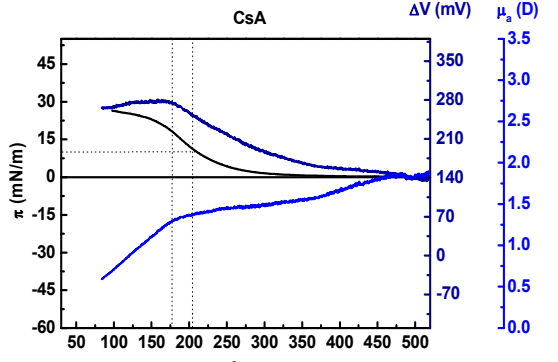

A ( $\AA^{2} /$ molecule $)$
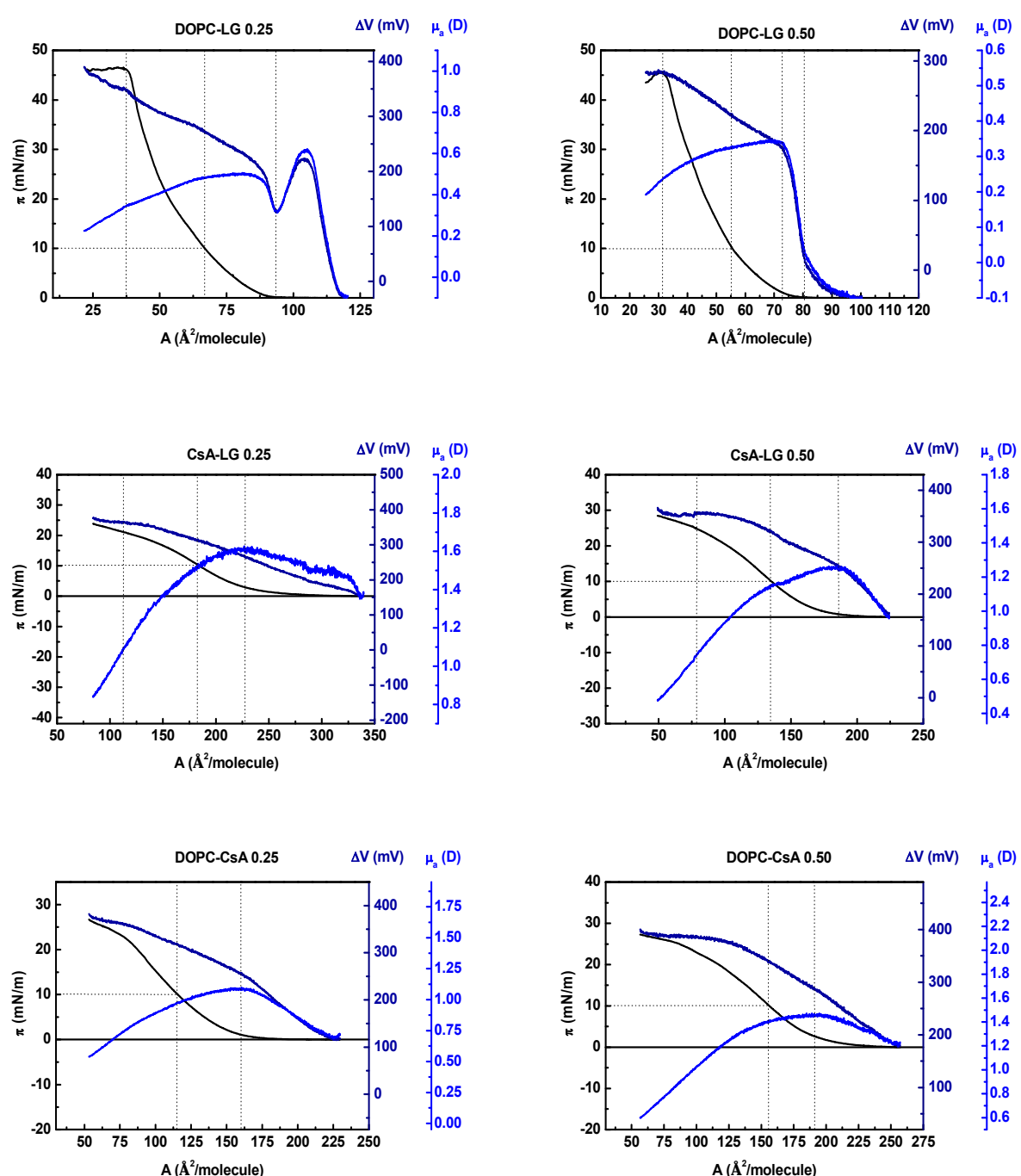
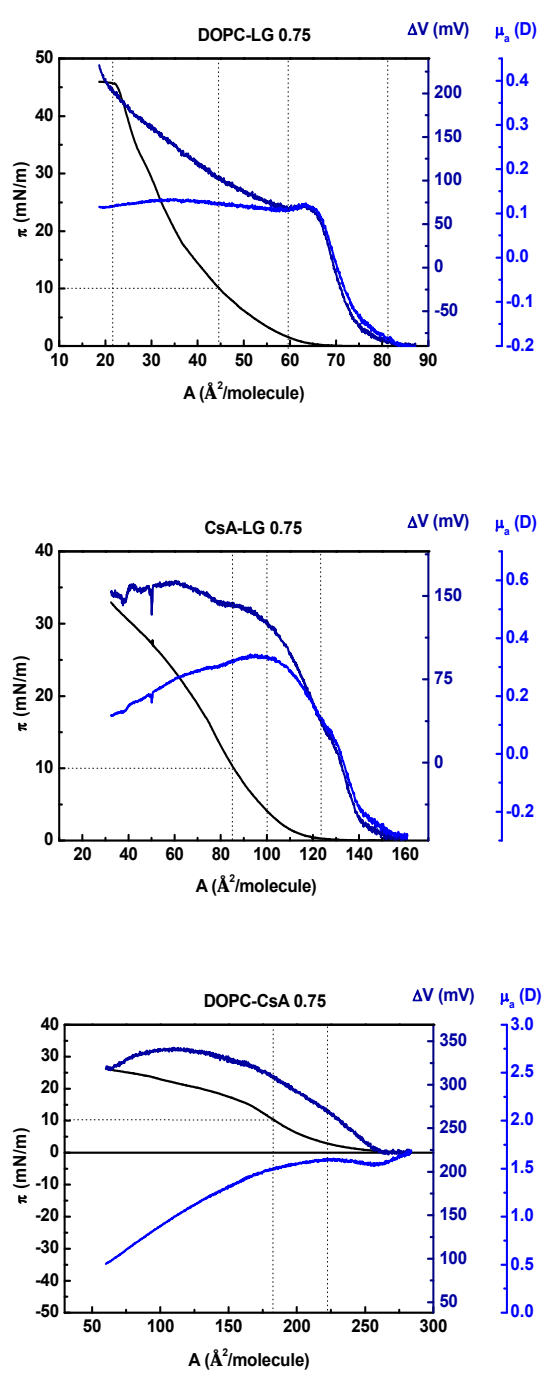

Figure S2. Surface pressure, $\pi-$, electric surface potential, $\Delta V-$, and apparent dipole moment, $\mu_{a}-$, area, $A$, isotherms for all monolayers studied. 


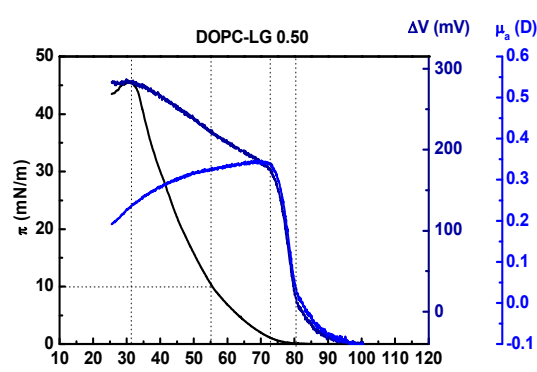

A $\left(\AA^{2} /\right.$ molecule $)$
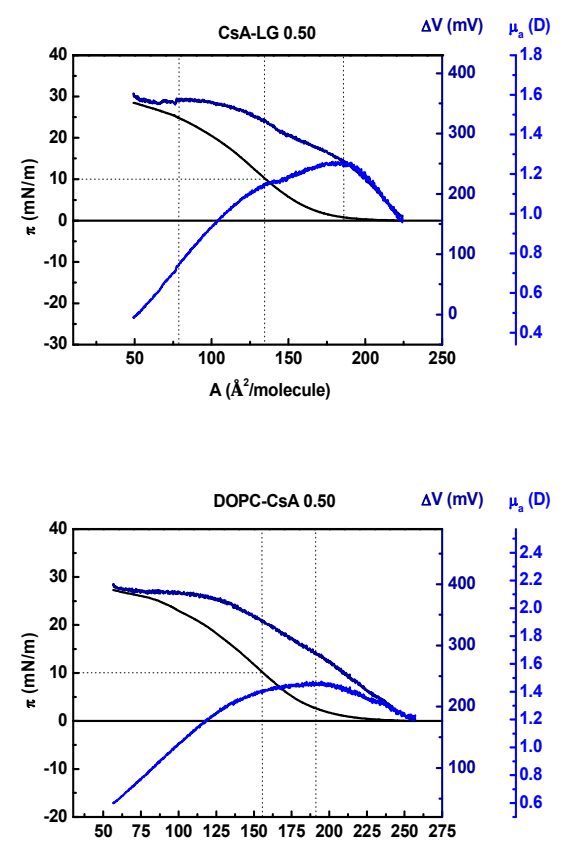
A $\left(\AA^{2} /\right.$ molecule $)$
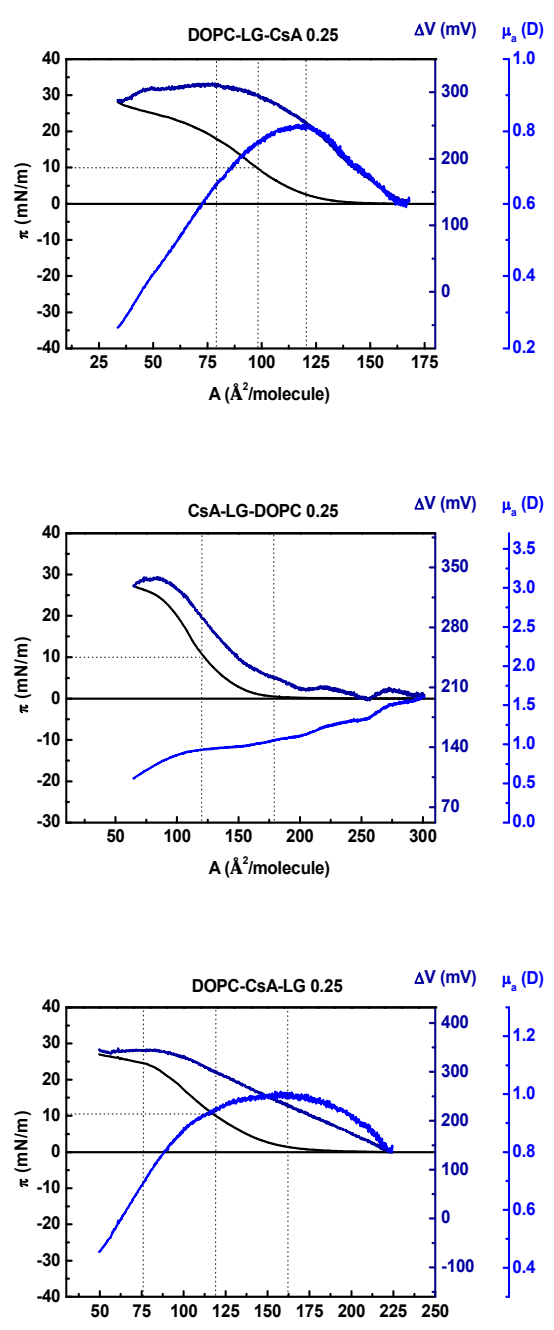

$A\left(\AA^{2} /\right.$ molecule $)$
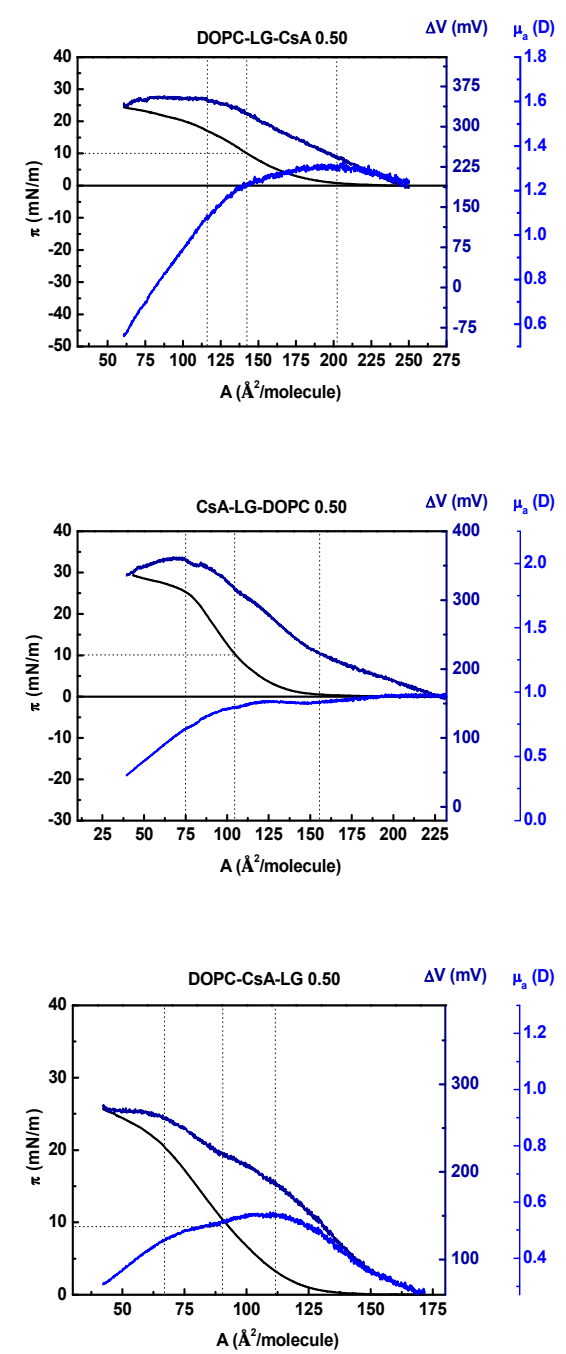
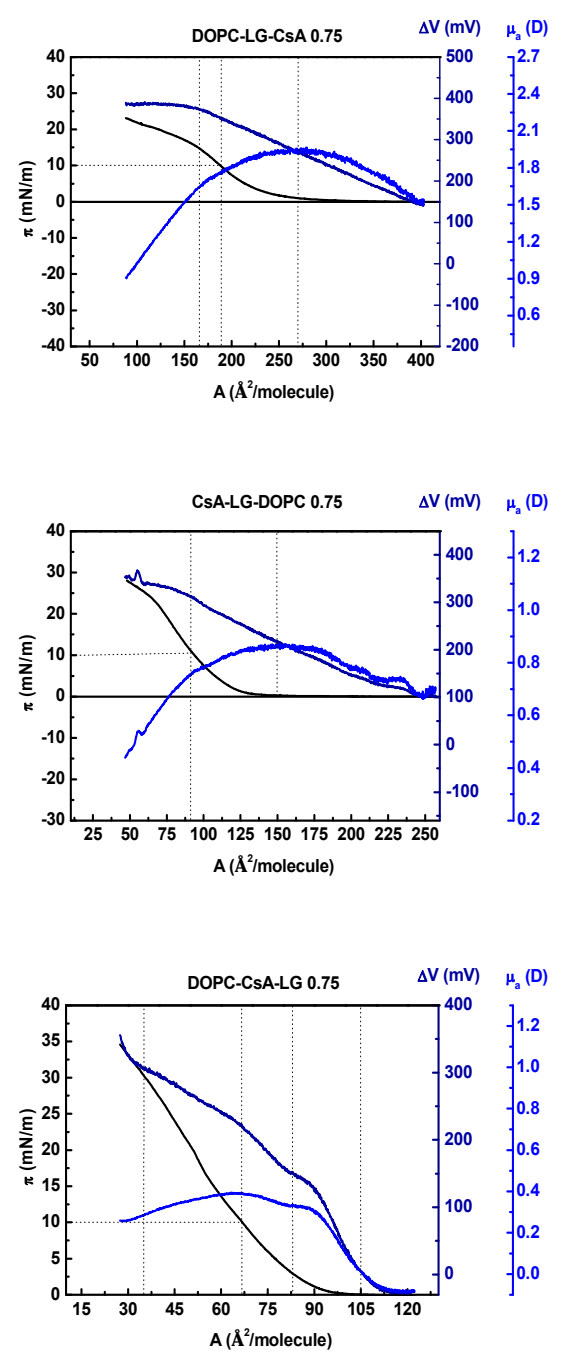

Figure S2. Surface pressure, $\pi-$, electric surface potential, $\Delta V$-, and apparent dipole moment, $\mu_{a}-$, area, $A$, isotherms for all monolayers studied (continued). 